Review

\title{
Refolding Technologies for Antibody Fragments
}

\section{Tsutomu Arakawa $^{1, *}$ and Daisuke Ejima ${ }^{2}$}

1 Alliance Protein Laboratories, 6042 Cornerstone Court West, San Diego, CA 92121, USA

2 Institute for Innovation, Ajinomoto Co. Inc., Kanagawa 210-8681, Japan

* Author to whom correspondence should be addresses; E-Mail: tarakawa@ap-lab.com;

Tel.: +81-858-550-9401; Fax: +81-858-550-9403.

Received: 20 January 2014; in revised form: 26 April 2014 / Accepted: 13 May 2014 /

Published: 23 May 2014

\begin{abstract}
Refolding is one of the production technologies for pharmaceutical grade antibody fragments. Detergents and denaturants are primarily used to solubilize the insoluble proteins. The solubilized and denatured proteins are refolded by reducing the concentration of the denaturants or detergents. Several refolding technologies have been used for antibody fragments, comprising dilution, dialysis, solid phase solvent exchange and size exclusion chromatography, as reviewed here. Aggregation suppressor or folding-assisting agents, including arginine hydrochloride, ionic liquids and detergents or denaturants at low concentrations, are included in the refolding solvent to enhance refolding yield.
\end{abstract}

Keywords: refolding; dialysis; dilution; C12-L-Glu; antibody; fragments

\section{Introduction}

Monoclonal antibodies occupy a large portion of therapeutic proteins [1]. Their smaller versions, e.g., scFv, are also intensively developed as more effective biopharmaceuticals [2,3]. Although gene constructs of these smaller antibody fragments are readily made [2,3], expression of folded fragments turns out to be rather difficult due to the non-native structures [4-6]. Whereas, they can be readily made in bacterial cells as inclusion bodies (IBs) [5,7,8]. Formation of IBs and refolding of the insoluble proteins have been, and still are, major obstacles in fully utilizing Escherichia coli cytoplasmic expression technologies. Various refolding procedures have been developed for therapeutic proteins and applied for antibody fragments [6,9-11]. Antibodies and many antibody fragments are composed of multiple domains, often connected by inter-domain disulfide bonds, 
which further complicates the already difficult refolding processes. The refolding process consists of solubilization, removal of solubilizing reagents and, for disulfide-containing proteins such as antibody fragments, oxidation of sulfhydryl groups. We have developed a novel refolding technology using an acylamino acid detergent, N-lauroyl-L-glutamate (C12-L-Glu) [12,13], and as reviewed in one of this series [14], based on two model proteins and scFv constructs. Here, we describe the various refolding technologies and compare with refolding using C12-L-Glu.

\section{Dilution Refolding}

One of the most conventional refolding protocols uses denaturants for solubilization and dilution for refolding. Below is the summary of a protocol developed by Buchner and Rudolph [15] for refolding of Fab fragment. IB solubilization was done with $6 \mathrm{M}$ guanidine hydrochloride $(\mathrm{GdnHCl})$ containing a reducing agent that reduces any disulfide bonds aberrantly formed in preparation of IBs or during refolding reaction. During the refolding process, an oxidizing agent, such as oxidized glutathione (GSSG), is incorporated. For example, the Fab was refolded by dilution of solubilized IBs into a solvent containing $0.4 \mathrm{M}$ arginine hydrochloride (ArgHCl), $2 \mathrm{mM}$ EDTA, $3 \mathrm{mM}$ dithioerythritol and $4 \mathrm{mM}$ GSSG. Inclusion of $\mathrm{ArgHCl}$ greatly enhanced the refolding yield of the Fab. In the above dilution refolding, the yield was only $16 \%$ in the absence of $\mathrm{ArgHCl}$ and greatly enhanced by the addition of $0.35-0.5 \mathrm{M} \mathrm{ArgHCl}$, reaching $\sim 40 \%$. This dilution refolding was found to be a slow reaction, requiring over $100 \mathrm{~h}$ incubation. $\mathrm{ArgHCl}$ may have slowed the refolding reaction, but perhaps helped structure rearrangement. Such structure rearrangement may prefer lower reaction temperature, which turned out to be true, as observed by an optimal temperature of $10{ }^{\circ} \mathrm{C}$. It should be noted here that presence of $E$. coli proteins in IBs does not appear to affect refolding yield, meaning that purification prior to refolding is not essential. A slow refolding and refolding enhancement by $\mathrm{ArgHCl}$ have been observed for scFv-exotoxin fusion protein [16], in which refolding of the scFv domain was a slow process and was strongly affected by the linker sequence between the scFv and exotoxin.

Ionic liquid can be used as a refolding assisting agent, just as is the case for ArgHCl. A majority of ionic liquids is made of organic cations (e.g., N,N'-substituted imidazolium, N-substituted pyridinium, tetraalkylated ammonium and tetraalkylated phosphorium) and either organic or inorganic anions [17]. The addition of ionic liquids in refolding solvents has resulted in aggregation suppression during refolding [18]. Whether a particular ionic liquid is a protein-denaturant or an aggregation suppressor appears to depend on its chemical structure and also the protein $[18,19]$. Effectiveness of ionic liquids as a refolding additive was tested using scFvOX ( $\mathrm{scFv}$ derived from anti-oxazolone antibody) [19]. The IBs were solubilized in $6 \mathrm{M} \mathrm{GdnHCl,} \mathrm{0.1} \mathrm{M} \mathrm{Tris,} \mathrm{pH} 8.0,100 \mathrm{mM}$ DTT and $1 \mathrm{mM}$ EDTA and buffer-exchanged into $4 \mathrm{M}$ GdnHCl. Refolding was initiated by rapid dilution into a solvent containing $2.5 \mathrm{mM}$ GSH, $0.25 \mathrm{mM}$ GSSG, $1 \mathrm{mM}$ EDTA, 0.1 M Tris, $\mathrm{pH} 8.0$ and ionic liquid. This diluted scFv solution was incubated for 4 days, reflecting the slow refolding process of scFv. As is true for many proteins, $\mathrm{ArgHCl}$ at 0.5 and $1 \mathrm{M}$ was effective as a refolding additive for this particular antibody fragment as well. Among the organic N'-methylimidazolium cations tested ( $\mathrm{Cl}$ as a counterion), those with long alkyl-chains (butyl and hexyl) were marginally effective. Those with short alkyl-chains (ethyl and propyl) showed enhanced refolding at optimal concentrations of $0.3-0.8 \mathrm{M}$. The dilution refolding used here resulted in significant precipitation in the absence of ionic liquids or ArgHCl. 
These ionic liquids were able to suppress scFv aggregation and precipitation during dilution refolding experiments, with the more hydrophobic long alkyl-chain cations more effective. However, these hydrophobic organic cations resulted in large reduction in protein stability, thus overwhelming their suppressive effects on protein aggregation: i.e., they solubilized the unfolded protein and could not promote refolding. Thus, it does appear that less hydrophobic short alkyl-chain organic cation-based ionic liquids at low to moderate concentrations $(0.3-0.8 \mathrm{M})$ are similar to $\mathrm{ArgHCl}$. One clear difference between $\mathrm{ArgHCl}$ and ionic liquids may be the concentration dependence, as the effect of $\mathrm{ArgHCl}$ normally increases with concentration in contrast to the optimal concentration exhibited by ionic liquids.

\section{Dialysis Refolding}

One of the research-scale refolding techniques is dialysis, to gradually remove the denaturants used.

Solubilization of insoluble proteins by such a denaturant as $\mathrm{GdnHCl}$, followed by reduction of $\mathrm{GdnHCl}$ concentration by dialysis, has been used by Tsumoto et al. [9] for the refolding of two scFv constructs. They used stepwise dialysis to gradually reduce $\mathrm{GdnHCl}$ concentration and to introduce disulfide-exchange reagents (e.g., $\beta$-mercaptoethanol and GSSG) and $\mathrm{ArgHCl}$, as reviewed in this special issue. Following a similar approach, Yuasa et al. [20] refolded scFv isolated from IBs in $3.5 \mathrm{M}$ $\mathrm{GdnHCl}, 500 \mathrm{mM}$ imidazole, $5 \mathrm{mM}$ dithiothreitol (DTT) at $\mathrm{pH}$ 7.4. The exact dialysis step used is as follows:

(1) Dialysis of solubilized scFv in $3.5 \mathrm{M} \mathrm{GdnHCl}, 500 \mathrm{mM}$ imidazole, $5 \mathrm{mM}$ DTT, pH 7.4 vs. $2 \mathrm{M}$ $\mathrm{GdnHCl}$ in the basic buffer containing $50 \mathrm{mM}$ Tris, $\mathrm{pH}$ 8.0, 0.2 $\mathrm{M} \mathrm{NaCl}, 1 \mathrm{mM}$ EDTA.

(2) Dialysis vs. $1 \mathrm{M} \mathrm{GdnHCl}, 0.4 \mathrm{M} \mathrm{ArgHCl}, 1 \mathrm{mM} \mathrm{GSSG}$ and $2 \mathrm{mM} \mathrm{GSH}$ in the above basic buffer (50 mM Tris, $\mathrm{pH} 8.0,0.2 \mathrm{M} \mathrm{NaCl}, 1 \mathrm{mM}$ ).

(3) Dialysis vs. $0.5 \mathrm{M} \mathrm{GdnHCl}, 0.4 \mathrm{M} \mathrm{ArgHCl}, 1 \mathrm{mM}$ GSSG and $2 \mathrm{mM}$ GSH in the above basic buffer.

(4) Dialysis vs. phosphate-buffered saline (PBS).

Details of this protocol are described in one of the articles in this special issue.

This dialysis refolding was successfully adapted to diabody refolding [21]. Diabody is essentially identical to scFv constructs except for shorter linkers. A scFv construct has a long linker sequence between $\mathrm{V}_{\mathrm{H}}$ and $\mathrm{V}_{\mathrm{L}}$ domains so that they form correct domain-domain interactions. When the linker sequence is shortened, $\mathrm{scFv}$ is no longer able to form intra-molecular domain-domain interactions and instead form inter-molecular domain-domain interactions, leading to formation of so-called diabody, or other, assemblies. Ortega et al. [21] used stepwise dialysis for refolding a diabody dissolved in $6 \mathrm{M}$ $\mathrm{GdnHCl}$. The $\mathrm{GdnHCl}$ concentration was slowly and gradually reduced to zero at constant ArgHCl concentration of $0.4 \mathrm{M}$ and varying concentrations of disulfide-exchange reagents. They identified the step that brings $\mathrm{GdnHCl}$ concentration from 2 to $1 \mathrm{M}$ as a critical step for high yield refolding of this particular antibody. 


\section{Solid Phase Refolding}

One of the major problems in protein refolding is off-pathway aggregation in solution, where protein molecules exhibit Brownian motion. If protein molecules are spaciously separated from each other during refolding, aggregation problem can be largely eliminated. Such a refolding system can be achieved on a solid phase, to which a protein molecule is non-covalently attached. The solid phases used are ion exchange resin, metal chelating resin and zeolite [22]. Protein folding may be interfered with, however, when the protein binds to the solid phase through multiple sites, as depicted in Figure 1. For example, a protein molecule may bind to the solid phase through multiple hydrophobic, electrostatic or van der Waals interactions (case A, B and C). A specific binding of affinity tag, e.g., non-natural his-tag, has an advantage in monovalent binding to the affinity resin, e.g., Ni-column (Figure 1, case D). Such an example is refolding of a scFv bound to Ni-chelating HisTrap column in $8 \mathrm{M}$ urea, $0.5 \mathrm{M} \mathrm{NaCl}, 5 \mathrm{mM} \beta$-mercaptoethanol, $\mathrm{pH} 8$ at different amounts of protein load [23]: low protein density on the column reduces protein-protein interactions in the bound state. While bound on the column, a gradient wash was performed from $8 \mathrm{M}$ to $0.5 \mathrm{M}$ urea in $\mathrm{pH} 8$ buffer, but in the absence of $\beta$-mercaptoethanol. When this gradient wash was done without addition of disulfide-exchange reagents, i.e., under air oxidation, the recovery of folded $\mathrm{scFv}$ was $20 \%$. Disulfide formation in the bound scFv was accelerated by the additions of $1 \mathrm{mM} \mathrm{GSH}$ and GSSG during the urea gradient. The influence of the timing of the addition during the gradient is plotted in Figure 2, revealing the importance of the addition of disulfide-exchange reagents. When they were added at higher urea concentrations, i.e., at the beginning of refolding to the early stage ( $4 \mathrm{M}$ urea), the yield was slightly enhanced (30\%-40\%). When added at the later stage of refolding ( 1 and $2 \mathrm{M}$ urea), little enhancement was obtained, perhaps due to misfolding that had taken place already. This observation again appears to reflect the complex folding process of $\mathrm{scFv}$ : i.e., there may be a critical step, in which air oxidation is favored or, alternatively, in which disulfide-exchange reagent is required for correct folding. After completion of gradient refolding, the bound $\mathrm{scFv}$ was removed from the column by imidazole. Although this specific protein binding and subsequent gradient refolding on solid phase may be effective, a major disadvantage is his-tag, which is unlikely to be acceptable as biopharmaceuticals.

Figure 1. Various binding modes for solid phase refolding. A: Two electrostatic interactions $(+,+)$; B: Electrostatic $(+)$ and hydrophobic ( $\mathbf{n})$ interactions; C: Two non-electrostatic interactions; D: His-tag affinity interaction.

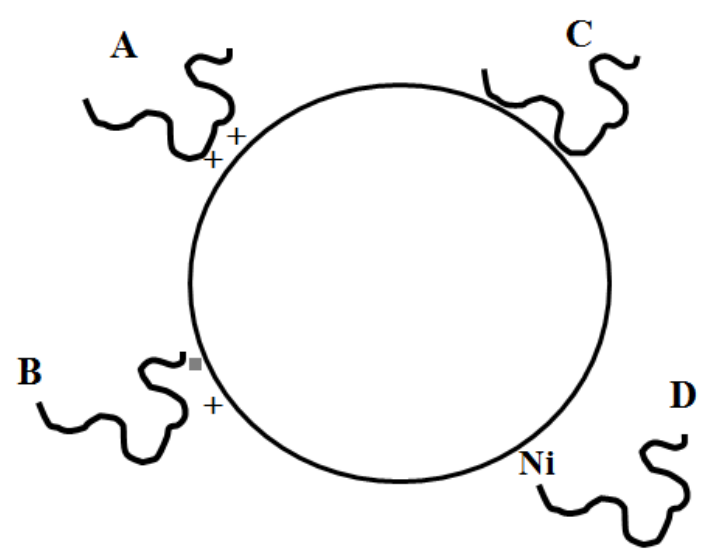


Figure 2. Refolding dependence on the timing of addition of disulfide exchange reagents.

Disulfide exchange reagents were added with different urea concentrations during refolding.

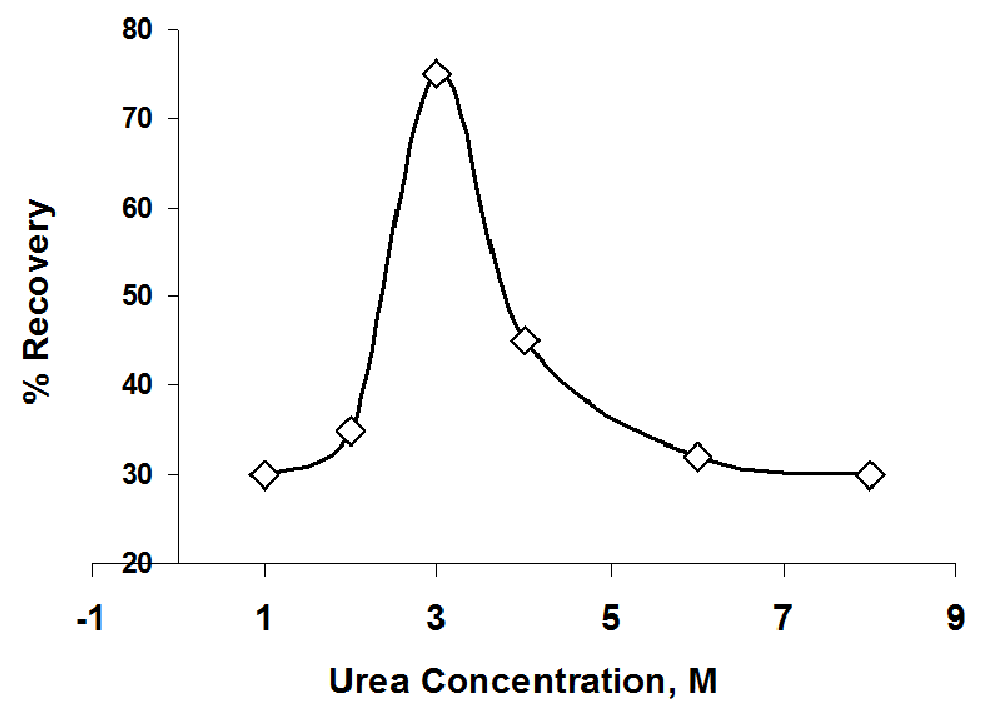

\section{SEC Refolding}

In size exclusion chromatography (SEC) refolding, the SEC column is used to exchange the solvent for protein molecules. In simple cases, for example, a fully unfolded protein in denaturant (e.g., $6 \mathrm{M}$ $\mathrm{GdnHCl}$ ) is injected into a column equilibrated with the refolding solvent: note that the refolding solvent must be suitable for the performance of SEC, e.g., acceptable viscosity or enough ionic strength and appropriate $\mathrm{pH}$ for minimal non-specific protein adsorption. After sample injection, the SEC column is developed with the refolding solvent. As the protein molecules migrate through the column, smaller denaturant molecules (e.g., $\mathrm{GdnHCl}$ ) migrate slower than the larger protein and hence are left behind. Thus, the denaturant concentration surrounding protein molecules gradually decreases as they move down the column, leading to refolding. Whereas in solution refolding, the motion of protein molecules is somewhat restricted by the column particles and hence aggregation can, in principle, be greatly reduced. In such a simple system, however, concentration gradient between the load and the column may be too steep to allow sufficient time for the unfolded scFv to slowly refold. A slower reduction in denaturant concentration can be achieved by establishing the denaturant gradient on the SEC column before loading the denatured sample, as depicted in Figure 3. An example of such an experiment was carried out by Yang et al. [24]. First, the SEC column was equilibrated with $0.2 \mathrm{M}$ urea, $\mathrm{pH} 7.5$ (refolding solvent). As depicted in Figure 3, the top 30\% portion of the column was used to establish a gradient of both urea concentration and $\mathrm{pH}$. The urea gradient from $8 \mathrm{M}$ (top) to $0.2 \mathrm{M}$ and the $\mathrm{pH}$ gradient from 2.0 (top) to 7.5 were formed. Upon establishing the gradient, the denatured $\mathrm{scFv}$ in $8 \mathrm{M}$ urea, 0.2 M DTT, $\mathrm{pH} 7.5$ was injected onto the top of the column that contained $8 \mathrm{M}$ urea, $\mathrm{pH}$ 2.0. Thus, the $\mathrm{scFv}$ protein was further unfolded by moving into a mobile phase containing $8 \mathrm{M}$ urea, $\mathrm{pH} 2.0$ (i.e., the sample $\mathrm{pH}$ was shifted from 7.5 to 2.0). Then, the unfolded scFv molecules flowed through descending urea concentration and ascending $\mathrm{pH}$, while leaving behind the reducing agent (DTT), $\mathrm{GdnHCl}$ and buffer species that migrate slower than $\mathrm{scFv}$, all these conditions leading to a more structure-forming (low urea concentration and neutral $\mathrm{pH}$ ) and oxidizing (no DTT) conditions. Finally, they exit the SEC column equilibrated with the mobile solvent containing $0.2 \mathrm{M}$ urea at $\mathrm{pH}$ 7.5. 
Figure 3. Size exclusion chromatography (SEC) refolding the top 30\% portion of the SEC column was used to establish the urea and $\mathrm{pH}$ gradient.

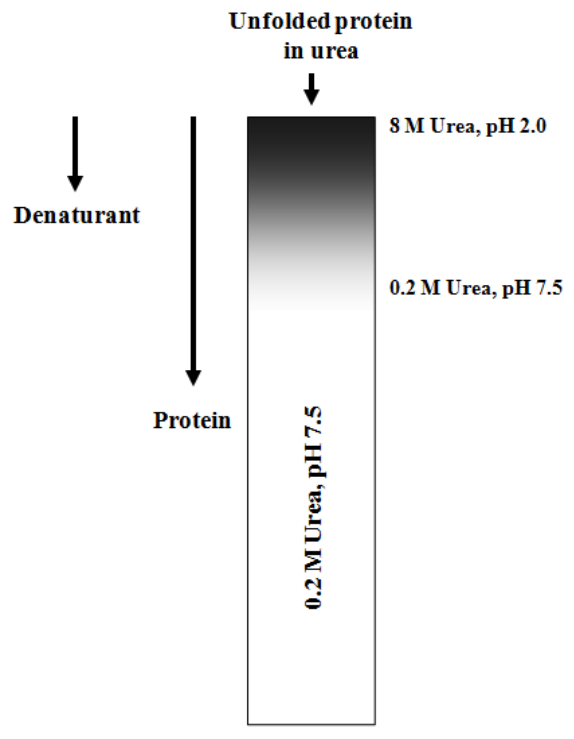

\section{Detergent Refolding}

Detergent that has been widely used for protein refolding is sodium N-lauroyl sarcosine (SLS) [25]. An attempt was made to refold three different $\mathrm{scFv}$ constructs expressed as IBs based on solubilization by SLS [5]. The IBs were solubilized with $2 \%$ SLS at pH 8-10. The solubilized scFv constructs were subjected to air oxidation at room temperature in the presence of $40-50 \mu \mathrm{M} \mathrm{CuSO}_{4}$ or $10 \mu \mathrm{M}$ sodium selenite: namely, oxidation of sulfhydril groups occurred in the presence of $2 \%$ SLS, at which the tertiary structure of the scFv is most likely unfolded, but non-native secondary structure is likely to be formed by the bound detergent. The SLS was removed from the scFv refolding solution by Dowex-1 anion exchange chromatography. Removal (or dissociation) of SLS was more efficient in the presence of $6 \mathrm{M}$ urea, suggesting strong binding of SLS to the scFv proteins. In the absence of $6 \mathrm{M}$ urea, the $\mathrm{scFv}$ proteins were also absorbed by the Dowex-1 due to the persistent protein/SLS complex structures. After removal of SLS, the scFv solutions were dialyzed against $\mathrm{pH} 8$ buffer to remove urea. The three steps, i.e., air oxidation in the presence of SLS, detergent removal and dialysis to remove urea, conferred refolding of the three scFv constructs tested.

We have developed a similar technology using a novel detergent, N-lauroyl-L-glutamate (C12-L-Glu) [12,13]. This detergent is unique in that it behaves as a strong protein solubilizer, as in other detergents used for protein refolding (e.g., SLS and sodium dodecylsulfate), but does not require anion exchanger for detergent removal. C12-L-Glu, as shown in Figure 4, is one of the acylamino acids. A number of acylamino acids are naturally present and hence can be physiologically metabolized. They are normally mild as a surfactant and possess various characteristics suitable as a household detergent [26]. Among various acylamino acids, C12-L-Glu appeared to be unique in certain detergent performances. As depicted in Figure 4, it is composed of C12 fatty acid and glutamic acid. Xia et al. [26] have extensively examined the properties of C12-L-Glu. Monosodium salt of N-lauroyl amino acids with five different amino acids (i.e., Gly, Glu, Ala, Val and Phe arranged in increasing hydrophobicity) showed decreasing CMC in this order, ranging $16 \mathrm{mM}$ for C12-Gly and $3 \mathrm{mM}$ 
for C12-L-Phe. As expected from micelle formation, these N-lauroyl amino acids all decreased the surface tension of water, with the magnitude in the reverse order. C12-L-Glu at $0.25 \%$ showed strong emulsifying power, a measure of mixing oil and water. It effectively stabilized the interface between oil and water. C12-L-Glu was also effective in dissolving hydrophobic organic compounds. Octyl-gallate and dodecyl-gallate are barely soluble in water. C12-L-Glu increased the solubility of both gallates by $\sim 10$-fold and $\sim 1000$-fold at 10 and $50 \mathrm{mM}$ : the strong effect at $50 \mathrm{mM}$ reflects the concentration above CMC (10.6 mM, 0.37\%) of C12-L-Glu [27].

Figure 4. Chemical structure of C12-L-Glu.

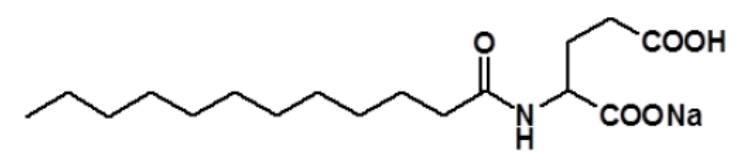

While effective as an emulsifier, C12-L-Glu appears to readily dissociate from proteins. Dissociation of C12-L-Glu from proteins was experimentally confirmed using model proteins [12]. C12-L-Glu at 2\% effectively caused denaturation of both bovine serum albumin (BSA) and interleukin-6 (IL-6). When the detergent concentration was reduced to $0.1 \%$, the native structure was restored, suggesting that the bound detergents have dissociated. Native gel analysis showed that the mobility of BSA or IL-6 was identical when the protein sample load on the native gel contained $0 \%, 0.1 \%$ and $0.2 \% \mathrm{C} 12-\mathrm{L}-\mathrm{Glu}$. It is thus likely that monomeric C12-L-Glu does not bind to the proteins, meaning that the detergent does dissociate from the protein when diluted below the CMC $(0.37 \%)$. The ability of C12-L-Glu to solubilize IBs was tested using E. coli expressed IL-6 and microbial transglutaminase [12]. These IBs were readily solubilized by $2 \%$ C12-L-Glu. Refolding was done by 20 -fold to 40 -fold dilution, which brings the C12-L-Glu concentration below $\mathrm{CMC}$. ArgHCl in the refolding solvent facilitated correct folding and suppressed protein aggregation. Simple dialysis or ion exchange chromatography was sufficient to make the final product free from C12-L-Glu. This technology was applied to solubilize and refold $\mathrm{scFv}$ [13]. In principle, $\mathrm{scFv}$ IBs were solubilized by $2.5 \% \mathrm{C} 12-\mathrm{L}-\mathrm{Glu}$ and diluted into refolding buffer containing both C12-L-Glu at low concentrations and ArgHCl. Unlike the refolding for IL-6 and transglutaminase, more complex refolding conditions were required for scFv, consistent with the fairly sophisticated dialysis refolding procedure [10] and the slow refolding [15] as described earlier. One of the scFv constructs tested showed an optimal yield using $5{ }^{\circ} \mathrm{C}$ incubation for $17 \mathrm{~h}$ followed by $23{ }^{\circ} \mathrm{C}$ incubation for $43 \mathrm{~h}$. A total of $60 \mathrm{~h}$ incubation is in accord with the long incubation used in denaturant refolding [15]. Optimal refolding using two different incubation temperatures suggest that refolding of scFv occurs through intermediate structures. One particular temperature may stabilize a specific intermediate structure. Based on this two-step incubation, the optimal refolding was obtained using a refolding solvent containing $0.8 \mathrm{M} \mathrm{ArgHCl}, \mathrm{C} 12-\mathrm{L}-\mathrm{Glu}$ at or above $0.15 \%$ and $1 \mathrm{mM} \mathrm{GSH}$ and GSSG. Thus, C12-L-Glu solubilized IBs at 2.5\% and facilitated refolding at low concentrations. These parameters, i.e., incubation time and temperature, $\mathrm{ArgHCl}$ and $\mathrm{C} 12-\mathrm{L}-\mathrm{Glu}$ concentration and oxidation condition, need to be optimized for refolding of each $\mathrm{scFv}$ or other antibody fragments. This was demonstrated with a different $\mathrm{scFv}$ construct. An optimal refolding solvent for this particular scFv was 0.1\% C12-L-Glu, $0.8 \mathrm{M} \mathrm{ArgHCl}$ and $1 \mathrm{mM}$ GSH and GSSG. However, incubation conditions were 
modified. In this case, three-step temperature changes appear to have given the best result: $18.5 \mathrm{~h}$ at $15^{\circ} \mathrm{C}$, $24 \mathrm{~h}$ at $23{ }^{\circ} \mathrm{C}$ and $4 \mathrm{~h}$ at $45^{\circ} \mathrm{C}$, in total over $46 \mathrm{~h}$ incubation.

It is interesting that incremental changes in the refolding temperature enhance the refolding yield. A similar dependence was also observed in other refolding systems. As described earlier, incremental reduction in $\mathrm{GdnHCl}$ concentration resulted in a greater yield in stepwise dialysis [10,21]. Solid phase refolding required optimal timing with regard to the addition of oxidizing agent. As schematically shown in Figure 5, the construct of antibody fragments consists of multiple domains: here $\mathrm{scFv}$ is made of two domains linked by a spacer sequence, i.e., a heavy chain variable domain, a linker and a light chain variable domain. Each domain (I and II in Figure 5) has a disulfide bond that should stabilize the domain structure. One possible mechanism may be stepwise folding: i.e., formation of intermediate structures followed by the formation of the native structure. Based on the temperature effects on recovery, it may be concluded that low temperature is favored in formation of the intermediate structure (Domain I in Figure 5) and high temperature accelerates the conversion of the intermediate to the native structure with correct domain-domain interactions. Such results may be consistent with different stability profiles between $\mathrm{V}_{\mathrm{H}}$ and $\mathrm{V}_{\mathrm{L}}$ domains [28]. This stepwise folding protocol by modulating incubation temperature may also be applicable to other refolding technologies, such as solubilization by denaturants or other detergents.

Figure 5. Schematic illustration of refolding course of scFv. Two black curves represent two domains (I, II). The gray line is a linker connecting Domain I and II.

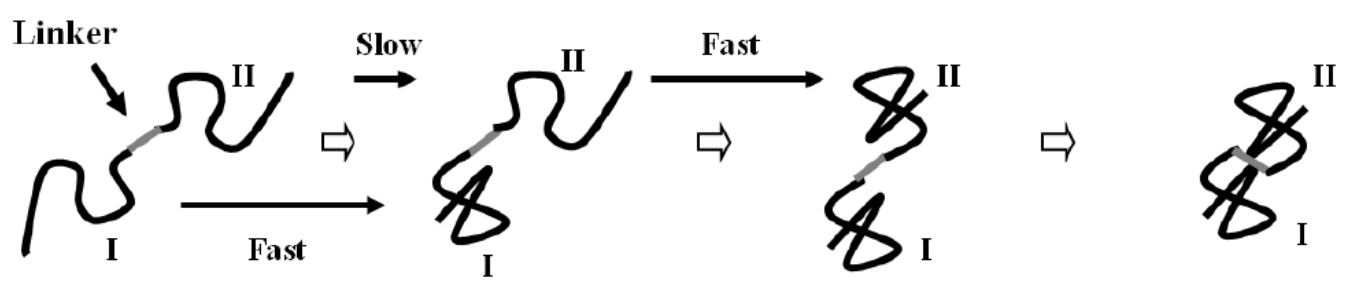

\section{Conclusions}

Antibodies and antibody fragments are clinically effective biopharmaceuticals. While antibodies can be readily made through mammalian expression technologies, production of non-natural antibody fragments is found rather difficult. In this review, various refolding technologies are summarized, as refolding appears to be the most effective for antibody fragments.

\section{Conflicts of Interest}

The authors declare no conflict of interest.

\section{References}

1. Yamada, T. Therapeutic monoclonal antibodies. Keio J. Med. 2011, 60, 37-46.

2. Shaefer, J.V.; Lindner, P.; Plückthun, A. Miniantibodies. In Antibody Engineering; Kontermann, R., Dübel, S., Eds.; Springer-Verlag: Berlin/Heidelberg, Germany, 2010; pp. 85-99. 
3. Plückthun, A.; Skerra, A. Expression of functional antibody Fv and Fab fragments in Escherichia coli. Meth. Enzymol. 1989, 178, 497-515.

4. Humphreys, D.P.; Glover, D.J. Therapeutic antibody production technologies: Molecules, applications, expression and purification. Curr. Opin. Drug Dev. 2001, 4, 172-185.

5. Anderson, D.C.; Reilly, D.E. Production technologies for monoclonal antibodies and their fragments. Curr. Opin. Biotechnol. 2004, 15, 456-462.

6. Kurucz, I.; Titus, J.A.; Jost, C.R.; Segal, D.M. Correct disulfide pairing and efficient refolding of detergent-solubilized single-chain Fv proteins from bacterial inclusion bodies. Mol. Immunol. 1995, 32, 1443-1452.

7. Arbabi-Ghahroudi, M.; Tanha, J.; MacKenzie, R. Prokaryotic expression of antibodies. Cancer Metastasis Rev. 2005, 24, 501-519.

8. Sun, W.; Xie, J.; Lin, H.; Mi, S.; Li, Z.; Hu, Z. A combined strategy improves the solubility of aggregation-prone single-chain variable fragment antibodies. Protein Expr. Purif. 2012, 83, 21-29.

9. Fursova, K.K.; Laman, A.G.; Melnik, B.S.; Semissotnov, G.V.; Kopylov, P.Kh.; Kiseleva, N.V.; Nesmeyanov, V.A.; Brovko, F.A. Refolding of $\mathrm{scFv}$ mini-antibodies using size-exclusion chromatography via arginine solution layer. J. Chromatogr. B 2009, 877, 2045-2051.

10. Tsumoto, K.; Shinoki, K.; Kondo, H.; Uchikawa, M.; Juji, T.; Kumagai, I. Highly efficient recovery of functional single-chain $\mathrm{Fv}$ fragments from inclusion bodies overexpressed in Escherichia coli by controlled introduction of oxidizing reagents-application to a human single-chain Fv fragment. J. Immunol. Methods 1998, 219, 119-129.

11. Fujii, T.; Ohkuri, T.; Onodera, R.; Ueda, T. Stable supply of large amounts of human Fab from the inclusion bodies in E. coli. J. Biochem. 2007, 141, 699-707.

12. Kudou, M.; Yumioka, R.; Ejima, D.; Arakawa, T.; Tsumoto, K. A novel protein refolding system using lauroyl-L-glutamate as a solubilizing detergent and arginine as a folding assisting agent. Protein Expr. Purif. 2011, 75, 46-54.

13. Kudou, M.; Ejima, D.; Sato, H.; Yumioka, R.; Arakawa, T.; Tsumoto, K. Refolding single-chain antibody (scFv) using lauroyl-L-glutamate as a solubilization detergent and arginine as a refolding additive. Protein Expr. Purif. 2011, 77, 68-74.

14. Arakawa, T.; Kita, Y.; Ejima, D. Refolding technology for scFv using a new dtergent, N-lauroylL-glutamate and arginine. Antibodies 2012, 1, 215-238.

15. Buchner, J.; Rudolph, R. Renaturation, purification and characterization of recombinant Fab-fragments produced in Escherichi coli. Bio/Technology 1991, 9, 157-162.

16. Brinkmann, U.; Buchner, J.; Pastan, I. Independent domain folding of Pseudomonas exotoxin and single-chain immunotoxin: Influence of interdomain connections. Proc. Natl. Acad. Sci. USA 1992, 89, 3075-3079.

17. Kragl, U.; Eckstein, M.; Kaftzik, N. Enzyme catalysis in ionic liquids. Curr. Opin. Biotechnol. 2002, 13, 565-571.

18. Summers, C.A.; Flowers II, R.A. Protein renaturation by the liquid organic salt ethylammonium nitrate. Protein Sci. 2000, 9, 2001-2008.

19. Lange, C.; Patil, G.; Rudolph, R. Ionic liquids as refolding additives: N'-alkyl and N'-(co-hydroxyalkyl) N-methylimidazolium chlorides. Protein Sci. 2005, 14, 2693-2701. 
20. Yuasa, M. Expression and structural characterization of anti-T-antigen single-chain antibodies (scFvs) and analysis of their binding to T-antigen by surface plasmon resonance. J. Biochem. 2013, 154, 521-529.

21. Ortega, C.; Herbet, A.; Richard, S.; Kersual, N.; Costa, N.; Pèlegrin, F.; Ducancel, F.; Couraud, J.-Y.; Navarro-Teulon, I.; Boquet, D. High level prokaryotic expression of anti-Müllerian inhibiting substance type II receptor diabody, a new recombinant antibody for in vivo ovarian cancer imaging. J. Immunol. Methods 2013, 387, 11-20.

22. Chiku, H.; Kawai, A.; Ishibashi, T.; Takehara, M.; Yanai, T.; Mizukami, F.; Sakaguchi, K. A novel protein refolding method using a zeolite. Anal. Biochem. 2006, 348, 307-314.

23. Guo, J.Q.; You, S.Y.; Li, L.; Zhang, Y.Z.; Huang, J.N.; Zhang, C.Y. Construction and high-level expression of a single-chain Fv antibody fragments for acidic isoferritin in Escherichia coli. J. Biotechnol. 2003, 102, 177-189.

24. Yang, X.; Hu, W.; Li, F.; Xia, H.; Zhang, Z. Gene cloning, bacterial expression, in vitro refolding, and characterization of a single-chain Fv antibody against PreS1(21-47) fragment of HbsAg. Protein Expr. Purif. 2005, 41, 341-348.

25. Lu, H.S.; Clogston, C.L.; Narhi, L.O.; Merewether, L.A.; Pearl, W.R.; Boone, T.C. Folding and oxidation of recombinant human granulocyte colony stimulating factor produced in Escherichia coli. Characterization of the disulfide-reduced intermediates and cysteine-serine analog. J. Biol. Chem. 1992, 267, 8770-8777.

26. Xia, J.; Nnanna, I.A.; Sakamoto, K. ProteinObased Surfactants. Synthesis, Physicochemical Properties, and Applications; Nnanna, I.A., Xia, J., Eds.; CRC Press: New York, NY, USA, 2001; pp. 75-122.

27. Ariki, R.; Hirano, A.; Arakawa, T.; Shiraki, K. Drug solubilization effect of lauroyl-L-glutamate. J. Biochem. 2012, 151, 27-33.

28. Wörn, A.; Plückthun, A. Mutual stabilization of $\mathrm{V}_{\mathrm{L}}$ and $\mathrm{V}_{\mathrm{H}}$ in single-chain antibody fragments, investigated with mutants engineered for stability. Biochemistry 1998, 37, 13120-13127.

(C) 2014 by the authors; licensee MDPI, Basel, Switzerland. This article is an open access article distributed under the terms and conditions of the Creative Commons Attribution license (http://creativecommons.org/licenses/by/3.0/). 